\title{
MOLECULAR DISSECTION OF THE SPORE KILLER ELEMENTS IN NEUROSPORA
}

\author{
David Rehard
}

\section{Dr. Patrick Shiu, Dissertation Supervisor}

\begin{abstract}
The role of selfish genetic elements in shaping the evolution of genomes (and subsequently species) has been a great subject of scientific interest for much of the last century. These selfish elements act at both the genetic and cellular level to insure that they are propagated within the genome, species, or both. Meiotic drive elements, which increase in number by destroying allelic counterparts, have been implicated in the extinctions of whole species and in the evolution of RNAi-mediated genome defense mechanisms. In fungi, several meiotic drive systems are known but not well understood. In the experiments described below, we attempt to characterize and differentiate the Spore killer $(S k)$ meiotic drive systems of the genus Neurospora as well as examine the defensive mechanisms that have arisen to prevent the spread of these and other selfish genetic elements.
\end{abstract}

The Neurospora Spore killer meiotic drive elements are believed to resemble other classic drive systems in their composition of two loci, a killer locus that creates drive for itself and a resistance locus that protects the killer from self-killing. The majority of my research herein has been a combined effort with Dr. Thomas Hammond on the mapping and characterization of the resistance locus in the Spore killer systems.

We began by utilizing the recently created Neurospora gene knockout library to create a series of 3-point testcrosses to map the location of a natural resistance gene in an $r(S k-2)$ strain. Testcross analysis narrowed the gene candidate field to just six genes. Next, knockout mutants were created for the remaining genes. Crosses of these mutants to $S k$ revealed that only one of the genes, NCU09151, confers resistance to Sk-2. We therefore identify NCU09151 as resistant to Spore killer $2\left[r s k^{r(S k-2)}\right]$. 
Next, we showed that when $r s k^{r(S k-2)}$ is inserted into a sensitive strain, resistance to $S k-2$ is granted to that strain, further solidifying the notion that NCU09151 is the resistant gene in the $r(S k-2)$ strains. Based on our crossing data, we also hypothesized that $S k-2$ must also use NCU09151 for its own resistance. Indeed, deletion of NCU09151 in Sk-2 lead to self-killing in a cross to a sensitive strain while introduction of $N C U 09151^{S k-2}$ to a sensitive strain granted resistance to $S k-2$.

After the resistance gene had been identified in the $S k-2$ system, we then asked whether the $S k-3$ system utilized the same gene for resistance. Gene knockout and transformation studies similar to those done on the $S k-2$ system revealed that $S k-3$ and $r(S k-3)$ strains also utilized the NCU09151 locus for resistance $\left[r s k^{S k-3}, r s k^{r(S k-3)}\right]$.

Sequence analysis of $r s k$ from both $S k-2$ and $S k-3$ revealed unique indel patterns specific to each Spore killer, solidifying the hypothesis that $S k-2$ and $S k-3$ are unique drive systems. This analysis, along with the observations from our prior experiments, allowed us to propose a killerneutralization model to explain how the killer molecule and the resistant protein might function in the two Spore killer systems.

Having identified the resistant component in the Neurospora Spore killer systems, we then wanted to tackle the question of how the drive systems are able to suppress meiotic silencing by unpaired DNA (MSUD). One possibility is that the Spore killers might harbor a dominant mutant gene that disrupts the MSUD system, thereby allowing unpaired genes to be expressed throughout meiosis. sad-p, a gene known to be required for proper MSUD is found within the Spore killer recombination block and is thus a prime candidate. Here we report that $s a d-p^{S k-2}$ is not a hypermorphic suppressor of MSUD. 